\title{
EDITORIAL
}

\section{A special issue on Biomedical Photonics}

\author{
Dan ZHU $(\bowtie)^{1,2}$, Shaoqun ZENG $(\bowtie)^{1,2}$, Valery V. TUCHIN $(\bowtie)^{3,4,5}$ \\ 1 Britton Chance Center for Biomedical Photonics, Wuhan National Laboratory for Optoelectronics, \\ Huazhong University of Science and Technology, Wuhan 430074, China \\ 2 Ministry of Education (MoE) Key Laboratory for Biomedical Photonics, Department of Biomedical Engineering, \\ Huazhong University of Science and Technology, Wuhan 430074, China \\ 3 National Research Saratov State University, Saratov 410012, Russia \\ 4 Institute of Precise Mechanics and Control RAS, Saratov 410028, Russia \\ 5 Interdisciplinary Laboratory of Biophotonics, National Research Tomsk State University, Tomsk 634050, Russia
}

(C) Higher Education Press and Springer-Verlag Berlin Heidelberg 2015

During the last decades, there have been significant developments in the field of biomedical photonics. We are pleased to present a special issue of Frontiers of Optoelectronics on Biomedical Photonics, which assembles one letter, three reviews, and eight original research articles.

Optical coherence tomography (OCT) has the ability to image tissues in vivo. Polarization-sensitive optical coherence tomography (PSOCT) is a functional extension of OCT. By measuring the polarization state of light reflecting or scattering back from tissue, PSOCT enables depth-resolved mapping of sample polarization information, such as phase-retardation and optical axis orientation. In this issue, Zhenyang Ding et al. gave a review of technology developments and biomedical applications of PSOCT. Fourier domain optical coherence tomography (FDOCT) provides cross-sectional images of tissue/material microstructures by spectral analysis of the low-coherence interference fringe pattern. Zhihua Ding et al. developed two novel systems with ultralong depth range. One is the orthogonal dispersive spectral domain optical coherence tomography (SDOCT), and the other is the recirculated swept source interferometer/OCT. No compromise between depth range and depth resolution is required in both systems. With their SDOCT system, Jian Gao et al. imaged mouse model with dorsal skin window chamber, they obtained a series of real-time OCT images and reconstructed 3D images of the specific area inside the dorsal skin window chamber by Amira.

Applications of two-photon microscopy (TPM) in pre-clinical and clinical study of human cancer were also reviewed in this issue. Based on the introduction of two-photon excitation, Jun Liu elaborated the advantages in imaging deep tissue and low photodamge of TPM for animal models and human samples. In addition, not only the applications of TPM on various aspects of tumor studies and clinical human skin biopsy, but also the technique development and the future directions were summarized. Wei Yan et al. presented a fluorescence endoscopic imaging system based on GRIN lenses using one-photon and two-photon excitation. Experimental results firstly showed that the system using two-photon excitation could implement dynamic fluorescence microendoscopic imaging and monitor the movement of blood flow beneath the skin in anesthetized mice except that it provide higher quality images with higher contrast and signal to noise ratio (SNR) than that using one photon excitation. It would be a useful tool for biologic investigations of small animals or plants in vivo.

Point spread function (PSF) engineering-based methods to enhance resolution and contrast of optical microscopes have experienced great achievements in the last decades. Each affords unique strengths in resolution, contrast, speed and expenses. Yue Fang et al. made a review to explore how PSF engineering generally could be used to break the diffraction limitation, and concluded that the common target of PSF engineering-based

Received April 20, 2015

E-mails: dawnzh@mail.hust.edu.cn, sqzeng@mail.hust.edu.cn, tuchinvv@mail.ru 
methods is to get a sharper PSF. Nonlinear effect and linear subtraction is the core techniques from the perspective of PSF reconstruction.

Shaosheng Dai et al. proposed a super-resolution infrared image reconstruction method using human vision processing mechanism (HVPM) by combining a mechanism of vision lateral inhibition with an algorithm projection onto convex sets (POCS) reconstruction. Experimental results demonstrate that the proposed method can significantly improve the visual effect of image, whose contrast and information entropy of reconstructed infrared images were significantly improved compared with traditional POCS reconstruction algorithm respectively.

As an only letter, Guo $\mathrm{He}$ et al. developed a high-resolution photoacoustic microscopy (PAM) to image in vivo blood vessels and capillaries of a mouse ear, even a single erythrocyte can be clearly imaged. There was a pair of accompanying venule and arteriole, whose detailed and further complicated branches can be clearly identified. And likely red blood cells (RBCs) arrayed one by one in microvasculature was also shown. The experimental results demonstrate that the high-resolution PAM has potential clinical applications for imaging of erythrocytes and blood vessels.

Laser speckle contrast imaging is known to be a powerful tool for blood flow mapping. Arkady S. Abdurashitov et al. described a simple algorithm based on histogram analysis of laser speckle contrast image for providing fast differentiation between macro- and microcirculations. The algorithm was successfully verified by the study of blood flow in rat cortex under functional activation.

Accurate diagnosis of within the group of different bronchopulmonary diseases is necessary in clinical practice. A. A. Bulanova et al. involved 20 healthy volunteers and 77 patients with bronchopulmonary diseases, including chronic obstructive pulmonary disease (COPD), bronchial asthma, pulmonary tuberculosis and community-acquired pneumonia. The absorption spectrum of exhaled air samples was recorded by an intra-cavity photo-acoustic gas analyzer based on photo-acoustic detector and $\mathrm{CO}_{2}$-laser with tuning range from 9.2 to $10.8 \mu \mathrm{m}$, which allowed making a preliminary diagnosis.

Integrating sphere technique is widely used to measure the total reflectance and transmittance of turbid sample, but the unavoidable light loss induces some measuring error. Xiewei Zhong et al. applied a convolution method based on the Monte Carlo simulation to obtain the reflectance and transmittance with rectangular light beam incident on turbid sample. The losses of light with various incident light beams (rectangular, circular and pencil light beams) were compared. In addition, the effects of optical properties and the rectangular incident light beam size on the light loss were also investigated.

The laser-induced tissue biological effect is relative to various factors. In this issue, Andrey V. Belikov et al. investigate diode laser under two continuous wave $(\mathrm{CW})$ or pulse modes to irradiate chicken meat samples using a quartz optical fiber either with a clear distal end (clear tip) or a distal end containing an opto-thermal converter (hot tip). The results demonstrated that diode laser with pulsed-mode resulted in a deeper crater in tissue, and width in collateral damage in soft tissue was larger than that with CW mode. In case of treatment with the hot tip, there were significant differences in crater depth and collateral damage width of chicken meat between diode lasers with CW and pulsed modes. For same average laser power, soft tissue treated with the hot tip had increased depth of crater in tissue and reduced width of collateral damage by comparing that treated with the clear tip. This work is useful for developing available laser treatment methods.

We would like to thank all the authors for their excellent contributions and the editors of Frontiers of Optoelectronics for inviting us to act as guest editors for this special issue.

Dan Zhu, Shaoqun Zeng, Valery V. Tuchin

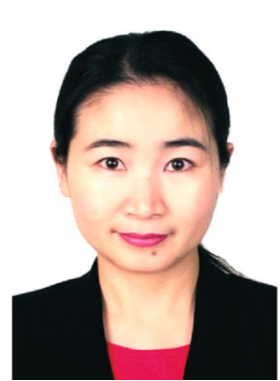

Dan Zhu received her B.S. degree in physics from Hubei University (1986), M.S. degree in physical electronics from Central China Normal University (1992), and Ph.D. degree in physical electronics from Huazhong University of Science and Technology (HUST), in 2001. After two years postdoctoral training at HUST, she joined as an Associate Professor in 2003 and a full Professor in 2007 in Biomedical Engineering with HUST. Currently, she is full Professor and Deputy Director of Division of Biomedical Photonics, Wuhan National Laboratory for Optoelectronics. She has authored more than 100 papers in the field of tissue optics, optical diagnosis and therapy principle and techniques. During the recent years, she has been focusing on optical clearing of tissue in vivo for improving optical imaging. She also severs as the Secretary General of Biomedical Photonics Committee of Chinese Optical Society, and a member of SPIE and OSA. She is editor member of Scientific Reports, Journal of Innovative Optical Health Science. 


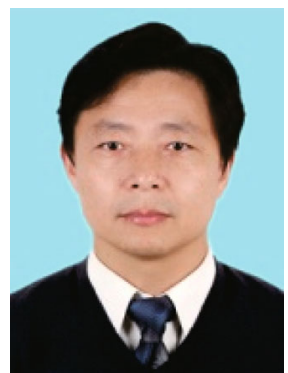

Shaoqun Zeng received his B.E. degree in optoelectronic engineering (1990), and Ph.D. degree in optoelectronics (1996) from Huazhong University of Science and Technology (HUST), Wuhan, China. Currently he is a Changjiang Professor of Britton Chance Center for Biomedical photonics, Wuhan National Laboratory for Optoelectronics. He is also a faculty member of the Department of Biomedical Engineering, HUST. He is also the Deputy Director for Research, Wuhan National Laboratory for Optoelectronics. His research interests include biomedical photonics, which involves developing novel optical principles and systems to explore the structure and functions of the neuronal networks, particular aims to improve the spatio-temporal resolution and throughput of optical imaging. For this purpose, he has investigated the basic physics of femtosecond laser pulse propagation, devices and multi-photon microscope systems to realize inertia-free beam scanning, signal processing techniques to record and recover action potential from Calcium fluorescence with single spike resolution, and thus initiate new applications in exploring the connections and functions of neuronal circuits. He has published around 90 papers, and hold more than 50 patents. He is a member of SPIE, OSA.

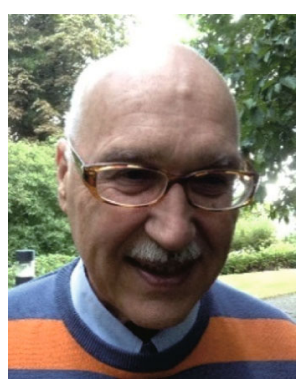

Valery V. Tuchin received his M.S. degree in radio-physics and electronics (1966), Ph.D. degree in optics (1974), and Dr.Sc. degree in laser physics (1982) from Saratov State University, Saratov, Russia. Currently he is a Professor and holds the Chair of Optics and Biophotonics of Saratov State University. He is also a Director of the ResearchEducational Institute of Optics and Biophotonics at Saratov State University and Head of Laboratory on Laser Diagnostics of Technical and Living Systems, Institute of Precise Mechanics and Control, RAS. His research interests include biophotonics, tissue optics, laser medicine, tissue optical clearing, and nanobiophotonics. He has authored more than 350 peer-reviewed papers, handbooks, monographs, text-books, tutorials, and book chapters, holder of more than 50 patents. He is a member of SPIE, OSA, and IEEE. He is a fellow of SPIE and has been awarded Honored Science Worker of the Russia (1999), SPIE Educator Award (2007), FiDiPro (Finland) (2011), and Chime Bell Prize of Hubei Province, China (2014). He is Editor-in-Chief of Journal of Biomedical Photonics \& Engineering and Associated Editor-in-Chief of Journal of Innovative Optical Health Science. 